\title{
Minimal model for calcium alternans due to SR release refractoriness
}

Inma R. Cantalapiedra, Enrique Alvarez-Lacalle, Angelina Peñaranda, Blas Echebarria ${ }^{1}$

Departament de Física. Universitat Politècnica de Catalunya, Av Dr. Marañon 50 (EPSEB), Barcelona, Spain

In the heart, rapid pacing rates may induce alternations in the strength of cardiac contraction, termed pulsus alternans. Often, this is due to an instability in the dynamics of intracellular calcium concentration, whose transients become larger and smaller at consecutive beats. This alternation has been linked experimentally and theoretically to two different mechanisms: an instability due to 1) a strong dependence of calcium release with sarcoplasmic reticulum (SR) load, together with a slow calcium reuptake into the SR or 2) to SR release refractoriness, due to a slow recovery of the ryanodine receptors (RyR2) from inactivation. The relationship between calcium alternans and refractoriness of the RyR2 has been more elusive than the corresponding SR Ca load mechanism. To study the former, we reduce a general calcium model, which mimics the deterministic evolution of a calcium release unit, to its most basic elements. We show that calcium alternans can be understood using a simple nonlinear equation for calcium concentration at the dyadic space, coupled to a relaxation equation for the number of recovered RyR2s. Depending on the number of RyR2s that are recovered at the beginning of a stimulation, the increase in calcium concentration may pass, or not, over an excitability threshold that limits the occurrence of a large calcium transient. When the recovery of the RyR2 is slow, this produces naturally a period doubling bifurcation, resulting in calcium alternans. We then study the effects of inactivation, calcium diffusion and release conductance for the onset of alternans. We find that the onset of alternans requires a well-defined value of diffusion while it is less sensitive to the values of inactivation or release conductance.

PACS numbers: 47.55.D-, 47.20.Ma, 47.11.-j, 68.03.Cd ionic models; ventricular arrhythmias; calcium alternans 
Cardiac alternans is a dysfunction that has been identified as a risk factor for cardiac arrhythmias. During alternans, beat-to-beat alternations in action potential duration (APD) appear alongside alternations in cytosolic calcium concentration. Despite been tightly coupled, voltage clamp experiments have shown that, often, the originating mechanism for alternans stems from an instability in calcium handling. Two alternative scenarios may explain this instability, either a slow reuptake of calcium after a release, or a long refractory period in release. In this paper we study the conditions for alternans due to refractoriness in calcium release, by reducing the dynamics of calcium to a simple two-variable model. The model includes a nonlinear dependence of release with calcium concentration and a slow recovery from refractoriness. This simple model presents excitability with a threshold that depends on the state of the ryanodine receptors (RyRs), i.e., calcium sensitive channels through which calcium is released to the cytosol. Calcium alternans appears as a bifurcation in which only one out of every two pulses is able to cross this excitability limit.

\section{INTRODUCTION}

Cardiac excitation starts at the sino atrial node as a periodic change in the myocytes' transmembrane potential, that then propagates along the atria and ventricles, inducing the contraction of the heart and the pumping of blood throughout the body. Failure to produce a proper excitation of cardiac tissue may results in life-threatening arrhythmias, as atrial (AF) or ventricular fibrillation (VF). A well-known precursor of VF is cardiac alternans $^{1}$, a disturbance in the normal rhythm of the heart characterized by a beat-tobeat alternations in the duration of the excited phase of the transmembrane potential, i.e., in the action potential duration (APD) and in the concentration of cytosolic calcium, which is the messenger that initiates contraction in cardiac myocytes. Since transmembrane voltage and calcium concentration transients are tightly coupled (through the action of the L-type calcium current and the sodium-calcium exchanger) beat-to-beat alternations in one of them transform into alternations in the other. Thus, besides unbalances in transmembrane currents that can give rise to APD alternans ${ }^{2}$, dysfunction in calcium handling can also be 
the reason behind the appearance of alternans. In effect, the persistence of contraction alternans in pacing protocols with periodic action potential waveforms ${ }^{3}$ indicates that this rhythm can be produced by beat-to-beat changes in the intracellular calcium response.

The originating mechanism that leads to a calcium transient instability is not completely understood $^{4-6}$. The calcium transient is initiated by an influx of calcium through the L-type calcium channel, that opens in response to a change in transmembrane potential. This rise in calcium concentration affects the opening probabilities of the calcium sensitive ryanodine receptors (RyR2) at the sarcoplasmic reticulum (SR), itself a membrane bound structure within the cell where most of the calcium is stored. Upon opening of the RyR2, calcium is released from the SR, increasing its concentration at the cytosol, and initiating contraction. The transient is finished either by the inactivation of the RyR2 channels or by the reduction of the calcium levels close to the RyR2, a volume of the SR called junctional SR. Finally, the action of the SERCA pump and the sodium-calcium exchanger, that pump calcium back to the SR and out of the cell, respectively, return calcium concentration to its basal value, so that it is ready to produce another transient.

Observation of SR calcium fluctuations during alternans ${ }^{7}$ suggested an important role of SR calcium load in the onset of alternans. The prevailing explanation is based on a steep relation between SR load and calcium release. This non-linearity, together with a slow reuptake due to SERCA, leads to an instability of regular calcium cycling at high SR calcium load and/or fast pacing rates, through a period-doubling bifurcation ${ }^{8}$. However, reports where cytosolic calcium alternans was found without appreciable change in SR calcium content $^{9,10}$, have opened the possibility of alternative mechanisms, such as SR-Ca release refractoriness. Optical mapping of healthy hearts by Wang et al ${ }^{11}$ shows that calcium alternans appears with or without variations in SR content, suggesting RyR2 refractoriness as responsible for their appearance at slower rhythms, sometimes followed by alternans due to SR load at faster stimulation. Experiments by Shkryl et al ${ }^{12}$ show that incomplete recovery of RyR2 from inactivation may result in alternans.

Restrepo et al showed ${ }^{13}$, using a detailed model of calcium handling that takes into account the interactions of many calcium release subunits, that calcium alternans could appear independently of the steepness of the release-load relationship. They proposed an alternative mechanism for alternans based on a beat-to-beat alternation in the number of refractory RyR channels. Other numerical studies have also highlighted the importance of 
RyR2 refractorines in order to explain experimental results: Lugo et $\mathrm{al}^{14}$ reproduced the experiments by Shkryl et $\mathrm{al}^{12}$ considering a slow recovery from inactivation in the dynamics of the RyR2. The analysis of a human atrial model by Chang et $\mathrm{al}^{15}$ shows that only the inactivation of RyR2 gives rise to alternans at slower pacing rates, as observed clinically in patients vulnerable to atrial fibrillation (AF).

In the cell, the global calcium signal is obtained from integrating many local calcium release events, or sparks. Refractoriness of release affects the likelihood of each of these events, themselves stochastic. The fact that refractoriness at a local level gives rise to a global period-doubling bifurcation was observed in an array of coupled stochastic, excitable elements $^{16}$. In ventricular cells, the link between local release events and global alternans has been shown to rely in three factors: randomness of Ca sparks; recruitment of a $\mathrm{Ca}$ spark by neighboring $\mathrm{Ca}$ sparks; and refractoriness of $\mathrm{Ca}$ release units (CaRU), in what has been termed as $3 \mathrm{R}$ theory ${ }^{17,18}$. Later on, this theory has been extended to include the effect of alternans due to a steep SR Ca load-release relation, showing that these mechanisms typically act synergistically ${ }^{19}$. Using a deterministic whole cell model, Alvarez et al ${ }^{20}$ showed that, depending on the kinetics of the RyR2, alternans may appear due to either SR load alternations, to a slow recovery of RyR2 from inactivation, or to a combination of both.

These results indicate that RyR2 refractoriness plays an essential role in the onset of calcium alternans and demands a better understanding of the particular effects of a change in its dynamics. Following this idea, in this paper we start from a modified ventricular myocite model ${ }^{20,21}$, able to reproduce calcium alternans by RyR2 refractoriness, and proceed to obtain a reduced model that contains just the essential elements that take part in this mechanism. This simplification facilitates an analytical treatment by reducing the mathematical description of the calcium transient to a nonlinear equation for the dyadic calcium concentration coupled to a relaxation equation for the number of recovered RyR2s, assuming fixed SR calcium concentration. While the release events are stochastic, in this paper we will consider the deterministic limit (corresponding to very fast diffusion in whole cell models where the global behavior of calcium is coordinated, or to a calcium release unit with a large number of RyR2). Even if this approximation will preclude us from giving a quantitative description of the transition in ventricular (or atrial) cells, the fact that it is a useful limit both at the global (cell) and local (CaRU) scale will allow us to obtain a deeper qualitative understanding on the dependence of the onset of alternans with different 

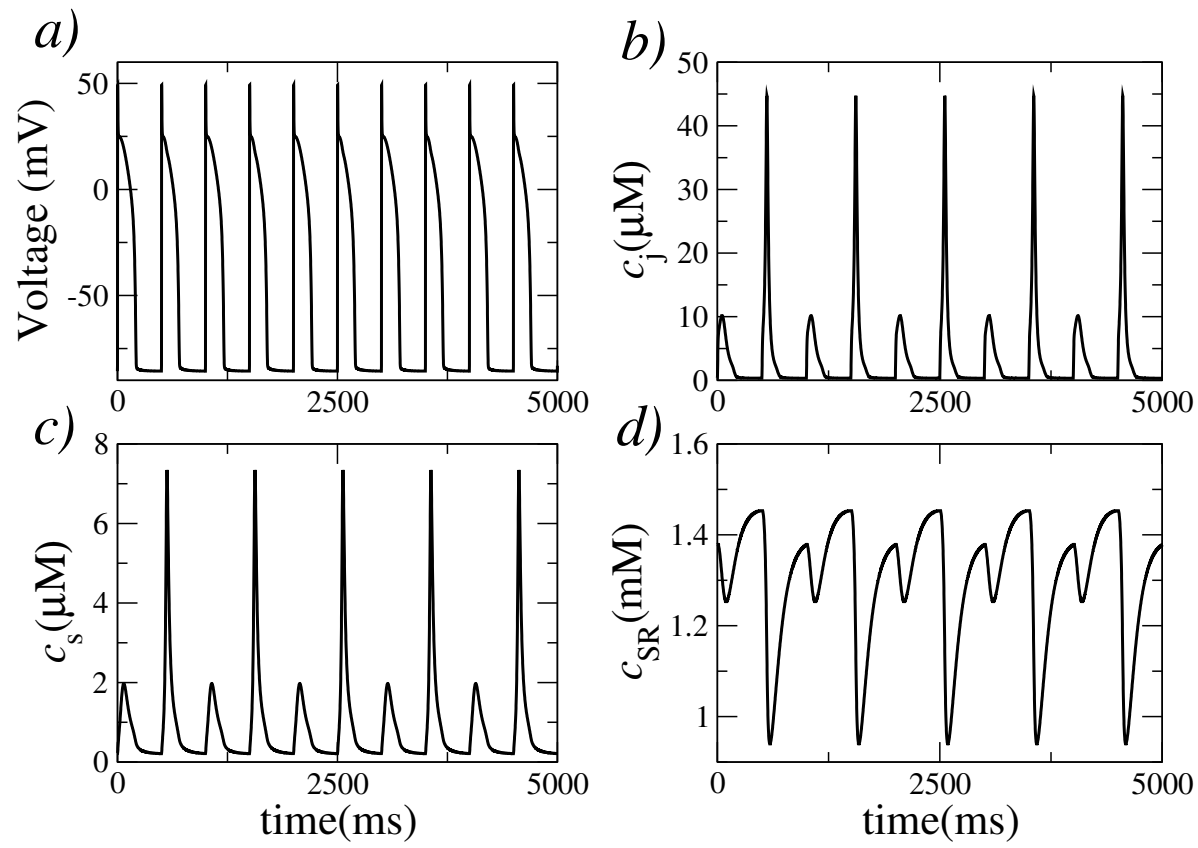

Figure 1. Time traces of a) transmembrane voltage, b) junctional, c) subsarcolemmal and d) SR calcium concentrations during alternans. The parameters are the same as in ${ }^{20}$, with $k_{a}=0.6 \mathrm{mM}^{-2}$, $k_{\text {im }}=0.013 \mathrm{~ms}^{-1}, k_{\text {om }}=0.06 \mathrm{~ms}^{-1}, k_{i}=0.5 \mathrm{mM}^{-1}$, and $T_{\text {period }}=500 \mathrm{~ms}$.

parameters involved in RyR2 kinetics and SR release.

The rest of the paper is organized as follows: in Section II we present the model for intracellular calcium handling. Section III is devoted to the analysis of the model and in Section IV we present the discussion and main conclusions of our work.

\section{MODEL AND APPROACH}

In Fig. 1 we show typical traces of transmembrane voltage and calcium during alternans, obtained with a rabbit ventricular myocyte model ${ }^{21}$. As in most whole cell models, calcium dynamics is splitted into different compartments of the cell given the large differences in concentration in the SR and in the cytosol. Even in the cytosol, concentrations are markedly different close to the membrane and further away from it. In this particular model there are four compartments: cytosol, sarcoplasmic reticulum (SR), subsarcolemma close to the cell membrane and junctional area (also called dyadic space) close to region of the membrane with L-type calcium channels (LCC). Calcium concentrations in each compartment are labelled as $c_{i}, c_{S R}, c_{s}$, and $c_{j}$, respectively. We use the same values of the parameters as $\mathrm{in}^{20}$, 


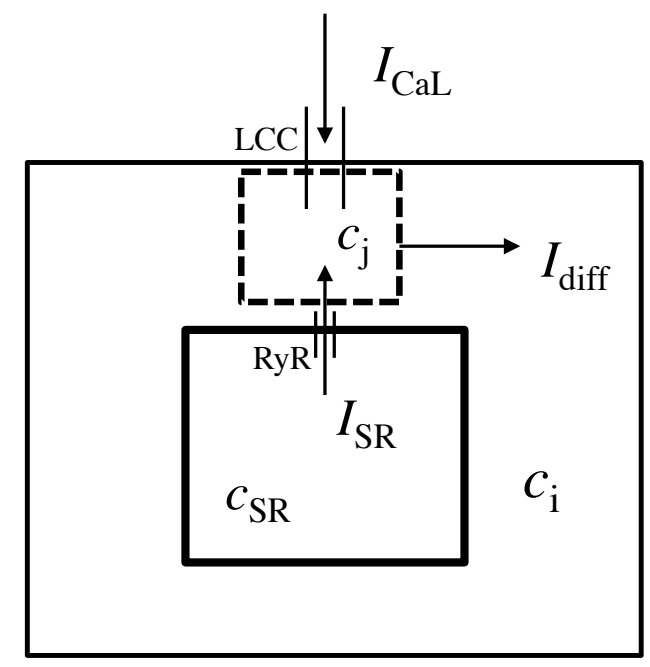

Figure 2. Calcium fluxes at the junctional space.

except for those related to RyR2 kinetics of activation, inactivation and recovery from inactivation that were varied with respect to the original model $i^{21}$ in order to obtain calcium alternans in the model as well as to reproduce the post-rest-potentation results described by Picht et al. ${ }^{9}$. As it was shown $i^{20}$, for these values of the parameters calcium alternans appears due to SR release refractoriness, stemming from a slow recovery from inactivation of the RyR2. This instability occurs due to the interplay of a slow recovery time scale of the RyR2 and a strongly nonlinear dependence of SR release with the number of recovered $\operatorname{RyR} 2^{4,23,24}$.

To better understand the effect of different parameters (diffusion, inactivation, release, etc) we will reduce the dynamics of calcium to a minimal model, that will suffice to reproduce calcium alternans. Although we do it for a specific model, the procedure we detail here will lead to the same simplified model for almost any single whole cell model of the literature, allowing us to test universal features of alternans due to RyR refractoriness in whole-cell calcium models. We will follow several steps:

- Decoupling of the dynamics of calcium at the junctional space from that of calcium at the other compartments. 
At the junctional space (see Fig. 2), calcium concentration $\left(c_{j}\right)$ is affected by fluxes coming from the extracellular medium $\left(I_{C a L}\right)$, from the SR $\left(I_{S R}\right)$, and from diffusion to the adjacent cytosol (or, depending on the model, subsarcolemmal) space $\left(I_{\text {diff }}\right)$ :

$$
\frac{d c_{j}}{d t}=I_{C a L}+I_{S R}-I_{d i f f}
$$

The SR release current is given by $I_{S R}=g_{r e l} P_{O}\left(c_{S R}-c_{j}\right)$, being $g_{r e l}$ the conductance of the RyR2 channels and $P_{O}$ the fraction of RyR2 channels that are in the open state. The diffusive current $I_{\text {diff }}=\left(c_{j}-c_{i}\right) / \tau_{\text {diff }}$ is just proportional to the calcium concentration difference between two compartments. The L-type calcium current is an inward current that depends on transmembrane voltage and calcium concentration.

To decouple the calcium dynamics at the junctional space from the other compartments we fix the calcium concentrations both at the SR $\left(c_{S R}\right)$ and at the cytosol space $\left(c_{i}=\right.$ $\left.c_{0}\right)$. Thus, this excludes the possibility of alternans due to SR calcium alternations ${ }^{7}$ and allows a strong control of the system given that we eliminate all homeostatic processes.

\section{- Simplification of the LCC type current.}

In order to retain a realistic but tractable problem, the behavior of the L-type calcium channels needs to be simplified. We will consider the L-type calcium current as an external stimulus, introducing a fixed amount of calcium during a given time, so

$$
I_{C a L}=\left\{\begin{array}{c}
I_{C a L}^{\max }, \quad \text { if } \bmod \left(t, T_{\text {period }}\right) \leq \Delta T \\
0, \quad \text { if } \bmod \left(t, T_{\text {period }}\right)>\Delta T
\end{array}\right.
$$

with $\Delta T=10 \mathrm{~ms}$. The L-type current described this way would correspond to the current through a LCC channel next to a cluster of RyR2 receptors. At the local level of the Calcium Release Unit (CaRU) where a cluster of RyR2 controls the release of calcium, LCC opening does trigger a roughly constant current of calcium. It is only the average of these stochastic opening among the thousands of LCC present in the cell, where some clusters open and others do not, which gives rise to a larger time-scale at the whole-cell level. Here we want to deal with a model which can be directly related to the behavior of a single CaRU in order to discuss its properties in a broader context. Thus, our model provides a description of how a CaRU would work 


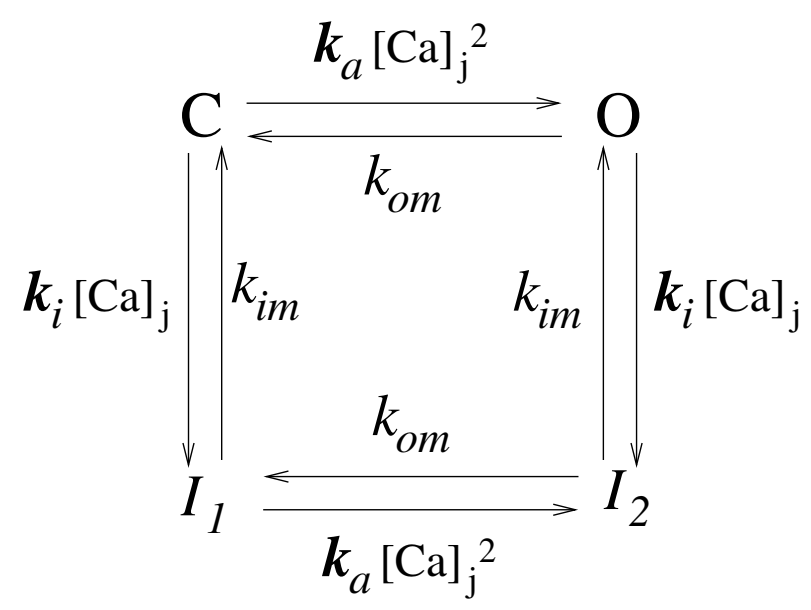

Figure 3. Representation of RyR2 gating dynamics ${ }^{25}$. The four markovian states of the RyR2 are $O$ (open), $I_{1}$ and $I_{2}$ (inactivated) and $C$ (close). The respective rates for transitions between states are represented. The recovery time is defined as $\tau=1 / k_{i m}$.

if, instead of 10-100 receptors in the cluster, thousands of them were present making the deterministic approximation correct.

Under this approximation, Eq. (1) becomes:

$$
\frac{d c_{j}}{d t}=I_{C a L}(t)+g_{r e l} P_{O}\left(c_{S R}-c_{j}\right)-\left(c_{j}-c_{0}\right) / \tau_{d i f f}
$$

so, with the previous simplifications, the dynamics of junctional calcium is only coupled to the dynamics of the RyR2 through the fraction of open RyR2 channels $P_{O}$.

- Simplification of the dynamics of the RyR2.

A schematic representation of the dynamics of gating of RyR2 channels $^{25}$ is shown in Fig. 3. The description of the RyR2 considers transitions among four states, one open $(O)$, one closed $(C)$, and two inactivated $\left(I_{1}, I_{2}\right)$, given by the probability rate equations:

$$
\begin{aligned}
& \frac{d P_{C}}{d t}=k_{i m} P_{I_{1}}-k_{i} c_{j} P_{C}-k_{a} c_{j}^{2} P_{C}+k_{o m} P_{O} \\
& \frac{d P_{O}}{d t}=k_{a} c_{j}^{2} P_{C}-k_{o m} P_{O}-k_{i} c_{j} P_{O}+k_{i m} P_{I_{2}} \\
& \frac{d P_{I_{1}}}{d t}=k_{o m} P_{I_{2}}-k_{a} c_{j}^{2} P_{I_{1}}-k_{i m} P_{I_{1}}+k_{i} c_{j} P_{C} \\
& \frac{d P_{I_{2}}}{d t}=k_{i} c_{j} P_{O}-k_{i m} P_{I_{2}}-k_{o m} P_{I_{2}}+k_{a} c_{j}^{2} P_{I_{1}}
\end{aligned}
$$


The dynamics of the RyR2 given by Eqs. (4)-(7) can be simplified using the fact that it possesses two invariant manifolds ${ }^{26}$, so its dynamics can be reduced to a two dimensional dynamical system for two variables $p$ and $q$, defined through $P_{C}=p q$, $P_{O}=q(1-p), P_{I_{1}}=p(1-q), P_{I_{2}}=(1-q)(1-p)$. By direct substitution it is easy to show that $p$ and $q$ satisfy the equations ${ }^{26}$

$$
\begin{aligned}
& \frac{d p}{d t}=k_{o m}(1-p)-k_{a} c_{j}^{2} p \\
& \frac{d q}{d t}=k_{i m}(1-q)-k_{i} c_{j} q
\end{aligned}
$$

From the previous definitions one can also write $p$ and $q$ as $p=P_{C}+P_{I_{1}}$ and $q=P_{C}+P_{O}$, being therefore $q$ the fraction of RyR2 that are in the close or open states, i.e. that have recovered from inactivation. These two equations represent a basic model compatible with the known physiological properties of the RyR2. The first one introduces the well known calcium-induced calcium-released nature of calcium transient. The second dictates the possibility of some sort of inactivation or termination of the RyR2.

Together with Eq. (3), this defines a three dimensional, nonautonomous dynamical system for the junctional calcium concentration and the state of the RyR2. In the limit of slow recovery times, compared with the opening time of the RyR2 $\left(k_{i m} \ll k_{o m}\right)$, we will consider that the opening of the RyR2 occurs almost instantaneously at the time scales of recovery. Thus, we can consider that Eq. (8) is in quasisteady state $d p / d t \simeq 0$, so

$$
p \simeq \frac{k_{o m}}{k_{o m}+k_{a} c_{j}^{2}}
$$

and, therefore

$$
P_{O}=q(1-p) \simeq q \frac{k_{a} c_{j}^{2}}{k_{o m}+k_{a} c_{j}^{2}}
$$

Then, using Eqs. (3), (9) and (11), we end up with a two dimensional system, given by:

$$
\begin{aligned}
\frac{d c_{j}}{d t} & =I_{C a L}(t)+g_{r e l} q \frac{k_{a} c_{j}^{2}}{k_{o m}+k_{a} c_{j}^{2}}\left(c_{S R}-c_{j}\right)-\left(c_{j}-c_{0}\right) / \tau_{\text {diff }} \\
\frac{d q}{d t} & =k_{i m}(1-q)-k_{i} c_{j} q
\end{aligned}
$$

These equations represent a basic model that includes all relevant physiological information needed to test and study the appearance of calcium alternans disconnected from SR alternation and homeostatic effects. 


\begin{tabular}{|c|c|c|}
\hline Parameter & Dimensional & Non dimensional \\
\hline RyR2 inactivation & $k_{i}=0.5 \mathrm{mM}^{-1} \mathrm{~ms}^{-1}$ & $\gamma=k_{i} / k_{i m} \sqrt{k_{o m} / k_{a}}=25$ \\
\hline RyR2 recovery from inactivation & $k_{i m}=0.002 \mathrm{~ms}^{-1}$ & \\
\hline RyR2 activation & $k_{a}=12 \mathrm{mM}^{-2} \mathrm{~ms}^{-1}$ & \\
\hline RyR2 closing & $k_{o m}=0.12 \mathrm{~ms}^{-1}$ & \\
\hline SR calcium concentration & $c_{S R}=500 \mu \mathrm{M}$ & $c_{S R} / \sqrt{k_{o m} / k_{a}}=5$ \\
\hline Cytosolic calcium concentration & $c_{0}=0.1 \mu \mathrm{M}$ & $c_{0} / \sqrt{k_{o m} / k_{a}}=0.001$ \\
\hline $\begin{array}{l}\text { Maximal L-type calcium current } \\
\text { conductance }\end{array}$ & $I_{C a L_{\max }}=50 \mu \mathrm{M} \mathrm{ms}^{-1}$ & $I_{C a L_{\max }} \sqrt{k_{a} / k_{\text {om }}} / k_{i m}=25$ \\
\hline RyR2 channel conductance & $g_{r e l}=0.556 \mathrm{~ms}^{-1}$ & $\alpha=g_{r e l} / k_{i m}=278$ \\
\hline $\begin{array}{l}\text { Diffusive time between dyadic and } \\
\text { cytosolic spaces }\end{array}$ & $\tau_{d i f f}=2 \mathrm{~ms}$ & $\beta=1 / k_{i m} \tau_{\text {diff }}=250$ \\
\hline
\end{tabular}

Table I. Standard values of the parameters in Eqs. (12), (13), taken from ${ }^{20}$.

Although in Table I we provide benchmark values for the parameters in these equations, wide uncertainties exist in most of them. In some cases, particularly for RyR2 conductance $g_{\text {rel }}$ and RyR2 inactivation $k_{i}$, broad disagreement exists even about their order of magnitude. The reason is that different order of magnitudes lead to different functional regimes of operation of the RyR2. A small conductivity $g_{r e l}$ requires a high probability of RyR2 opening making inactivation an unrealistic feature. On the other hand, a large conductance $g_{r e l}$ leads necessarily to a relevant level of inactivation, otherwise the calcium concentration in the junctional volume would reach unrealistic values. Another important parameter is $\tau_{\text {diff }}$, which indicates the effective diffusion, basically controlled by the level of protein crowding in the cytosol and the level of calcium buffer.

To eliminate some of the parameters we will further normalize junctional calcium concentration $c_{j}$ by $\sqrt{k_{o m} / k_{a}}$ and time by $\tau=1 / k_{i m}$. Then the two equations to be analysed are:

$$
\begin{aligned}
\frac{d c_{j}}{d t} & =I_{C a L}(t)+\alpha q\left(\frac{c_{j}^{2}}{1+c_{j}^{2}}\right)\left(c_{S R}-c_{j}\right)-\beta\left(c_{j}-c_{0}\right) \\
\frac{d q}{d t} & =1-q-\gamma c_{j} q
\end{aligned}
$$

The adimensional parameters are related to different features of the dynamics: $\alpha$ to the 

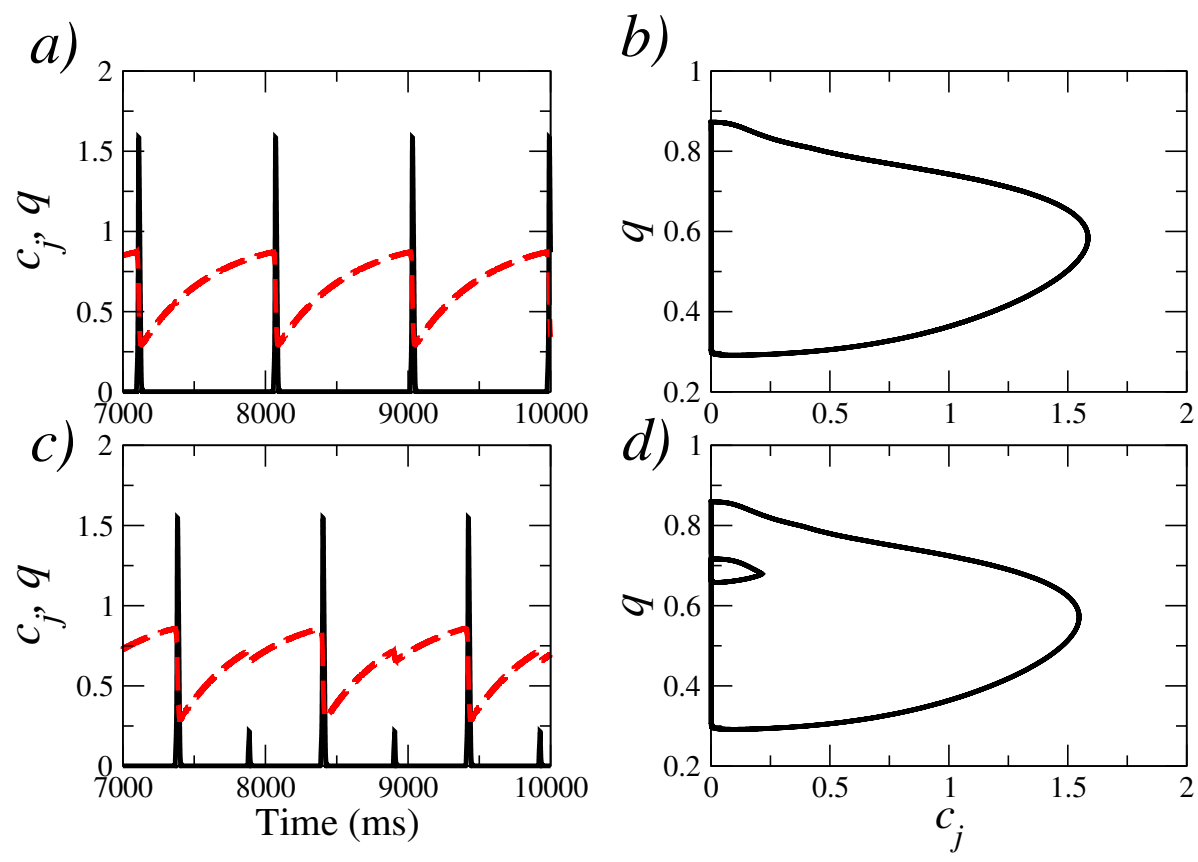

Figure 4. a) Evolution as a function of time of normalized junctional calcium $\left(c_{j}\right.$, in black solid line) and RyR2 recovery variable ( $q$, in dotted red line) for a pacing of the LCC at $T_{\text {period }}=960$ ms. b) The same evolution in phase space $c_{j}-q$, showing the standard calcium transient. c) and d) Appearance of calcium alternans with periodicity 2:1 where a large transient alternates with a small transient occurring at $T_{\text {period }}=505 \mathrm{~ms}$.

release strength of the RyR2, $\beta$ to difussion and $\gamma$ to the relevance of the inactivation (or termination process) of the receptor. Our goal is to fix the rest of parameters, which are reasonably well-known, and vary $\alpha, \gamma$ and $\beta$ from their standard values, shown in Table I, in order to test the effects on the dynamics.

\section{ANALYSIS OF THE MODEL}

\section{A. Calcium transient and 2:1 alternation}

The evolution of calcium $c_{j}$ in the junctional space and the recovery variable $q$ given by Eqs. (14), (15) shows the standard transient calcium behavior at long stimulation periods (Figs. 4a and b). LCC triggers the calcium-induced calcium-release opening of the RyR2 that results in a sharp increase in the level of junctional calcium. Upon closing of the LCC, the release of calcium finishes due to the inactivation of the RyR2 opening. The variable 
$q$ drops, closing the release (cf. Eq. (11)), and calcium is then diffused away, getting back to the basal level fixed in the model at $c_{o}$. Simulations of the model also show that an increase of the pacing frequency leads to the well-known 2:1 bifurcation with alternation of the strength in the calcium transient from one beat to the next (Fig. 4c and d). In this regime at a given beat the calcium transient is large while it becomes small at the following beat.

This is the same behavior as observed in the global whole cell model described in ${ }^{20}$ and the general mechanism is well understood. If, at a given beat, the number of recovered RyR2, $q$, is large, then, given the nonlinear relation between the calcium release strength and the number of recovered RyR2, release is also very large. However, this drives the inactivation of the receptors in the cluster, i.e., $q$ drops dramatically for this release. Provided that the recovery from inactivation is slow enough, at the next beat the number of recovered receptors is low. This produces a small release and also small inactivation with $q$ not dropping as much as in the previous beat. At the next beat the receptors are then fully recovered, starting the same process all over again.

While the general mechanism is clear, the details of the non-linear release dependence on $q$ are not completely understood. Formally, the release depends linearly on $q$ and it is only through the nonlinearity of the calcium-induced calcium-release process that a nonlinear interaction is generated. Following this line of thought, the relevance of the slow time scale of inactivation $\gamma$ is clear; a fast recovery will make this type of alternation impossible. On the other hand, the possible effects and relevance of diffusion $\beta$ and strength of release $\alpha$ is not clear given that their influence, if any, should be directly linked to the nonlinear properties of the release.

We proceed to pursue the analysis in two directions. First, we study the stability of the period one and period two orbits and test if there is any other type of behavior present in the model, such as different periodicities or more chaotic behavior. Second, we try to understand the nonlinear process that leads to 2:1 alternation, and probably to other types of periodicities, which should allow us to understand how diffusion or release strength may play a role in the alternans bifurcation. 


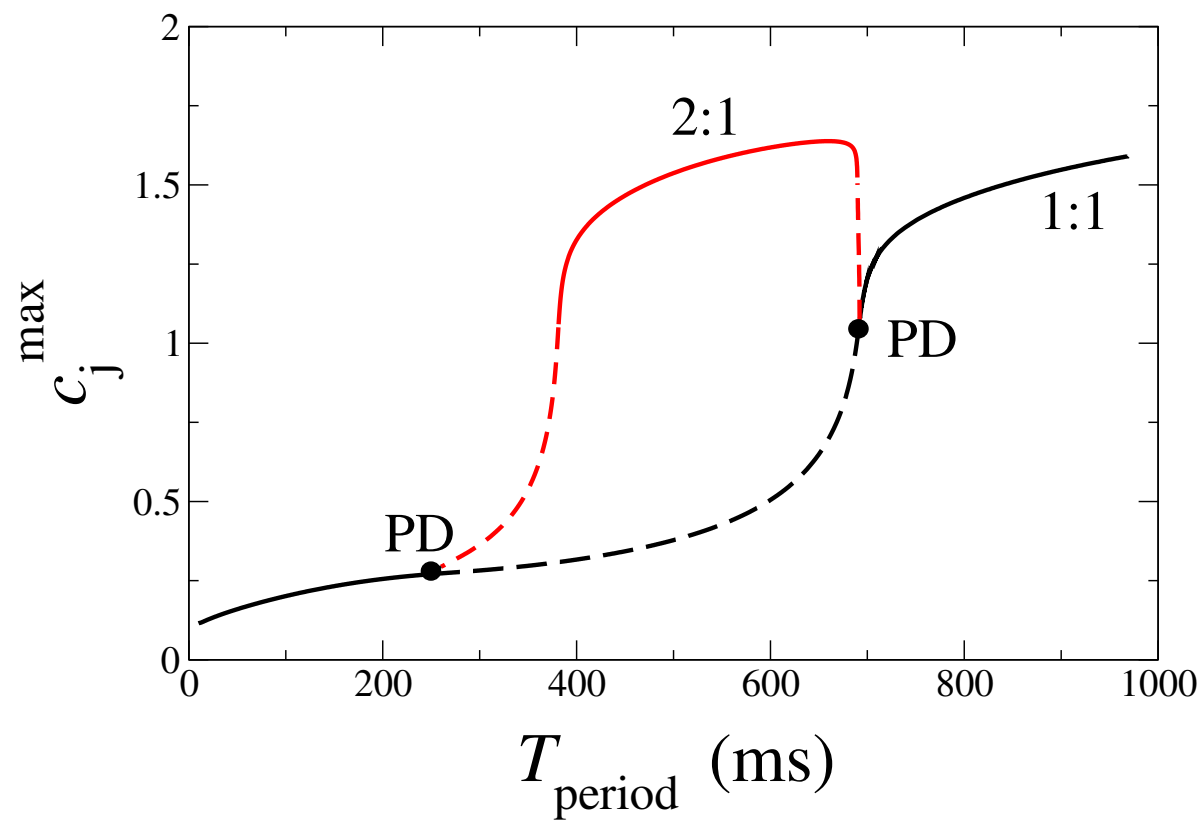

Figure 5. Bifurcation diagram. Stable solutions are indicated with black (1:1 branch) and red (2:1 branch) solid lines and unstable solutions with black and red dashed lines. The presence of a period-doubling bifurcation is indicated by PD. The values of the nondimensional parameters are: $I_{C a L}=25, \alpha=278, \beta=250, c_{S R}=5$ and $\gamma=25$.

\section{B. Bifurcation diagrams and higher order periodicities}

In order to find different behavior and understand the alternation process we first study the stability of the period one (1:1) and period two (2:1) solutions. We use the continuation software $\mathrm{AUTO}^{27}$, as implemented in XPPAUT ${ }^{28}$. Since this software only allows continuation of orbits in autonomous dynamical systems, we have converted our nonautonomous system given by Eqs. (14), (15) into an autonomous system, expressing the forcing $\left(I_{C a L}(t)\right)$ in terms of periodic functions. The details are given in Appendix A. Continuation techniques allow the analysis of the evolution of asymptotic solutions in a system of ODE when a parameter is varied. They also possess the important advantage of allowing the computation of unstable solutions and, thus, indicate a change in the type of stable solution.

A typical bifurcation diagram, plotting peak calcium concentration $c_{j}$ as a function of the stimulation period $T_{\text {period }}$, is shown in Fig. 5. The period one orbit, stable at large periods, becomes unstable through a period doubling bifurcation (PD) to a branch of period two solutions, as expected. This solution becomes unstable at lower stimulation periods until 

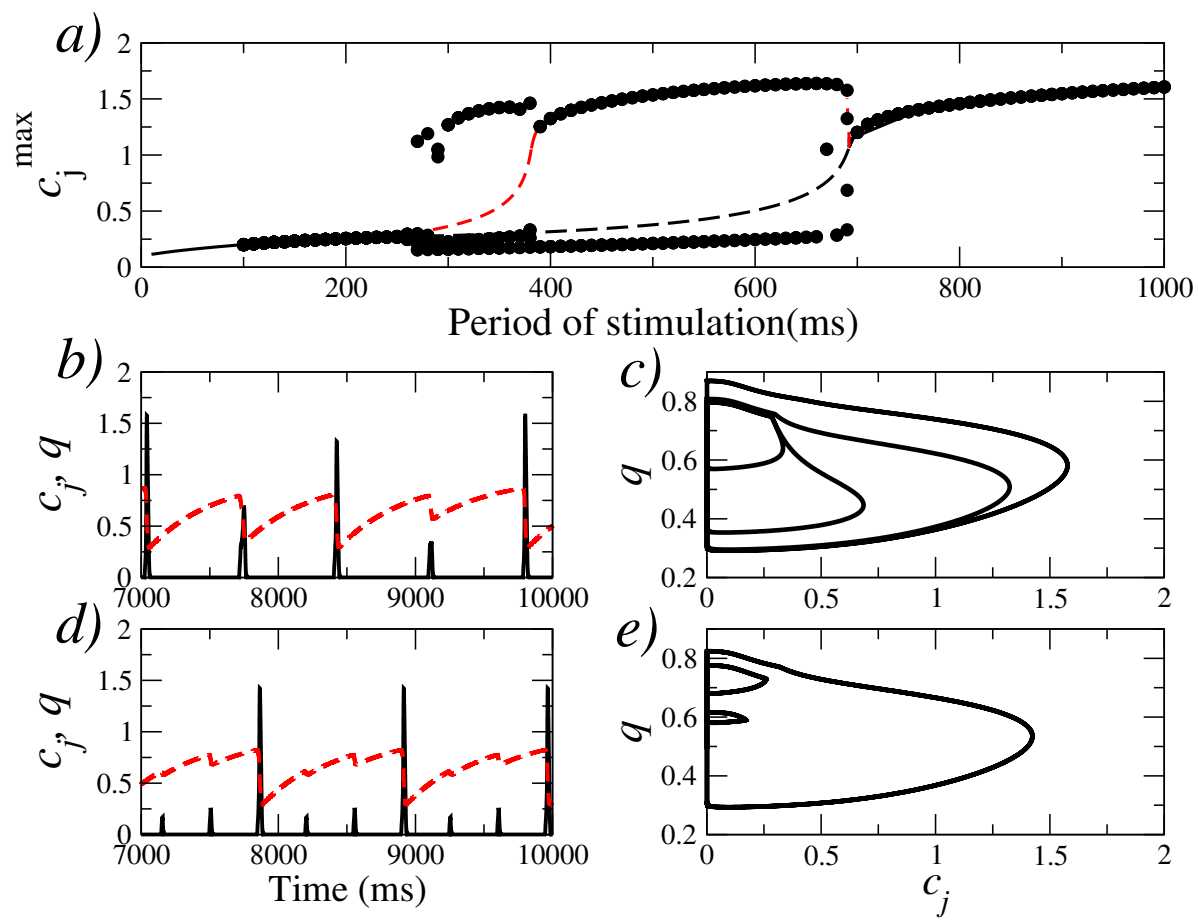

Figure 6. a) Bifurcation diagram as a function of pacing period $\left(T_{\text {period }}\right)$. The black dot points corresponds to the maximum value of $c_{j}$ during five beats, obtained from numerical integration of Eqs. (14), (15). The solid (dashed) lines corresponds to the stable (unstable) solutions in Fig. 5. In the lower panels we show the evolution of the variables $c_{j}$ with black solid line and $q$ with red dashed line, and the corresponding phase portrait, for different pacing periods: b) and c) $\left.T_{\text {period }}=290 \mathrm{~ms}, \mathrm{~d}\right)$ and e) $T_{\text {period }}=690 \mathrm{~ms}$.

the period one solution regains stability at very high frequencies with low $c_{j}$ peak values. Fig. 6a shows the solutions found by numerical integration on top of the bifurcation diagram presented in Fig. 5. As expected from the bifurcation diagram, the instability of the period one orbit gives rise to a period two orbit (alternans) at larger frequencies. At lower periods, in the region where the period 2 orbit is unstable, we observe a whole set of different complex dynamics.

The presence of complex and multiple bifurcations and instabilities from 2:1 alternations as pacing frequency increases agrees with the appearance high-order periodicities, and even of rather chaotic behaviour, observed in full models $\left(\mathrm{see}^{20}\right)$. We show in Fig. 6 typical examples of the more complex dynamics observed at periods where the period 1 and 2 orbits are unstable. We observe, for instance, period three orbits (Figs. 6d,e) and more irregular 

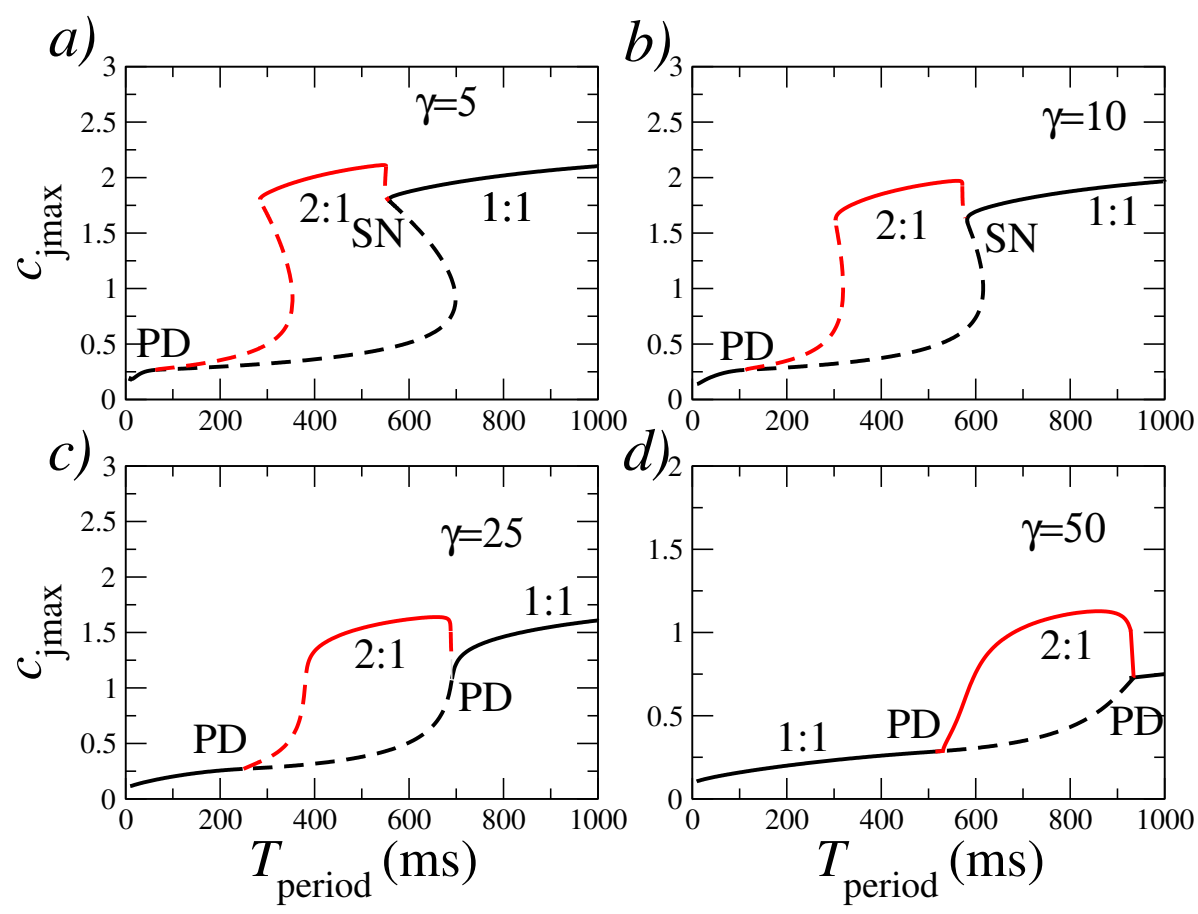

Figure 7. Bifurcation diagrams as a function of the period of stimulation for different values of the strength of the inactivation, $\gamma=5,10,25$ and 50. Solid line corresponds to the stable solution of the period one solution (black) or alternans (red) and dashed lines to the unstable ones. For lower values of $\gamma$ the period-doubling (PD) bifurcation transforms to a Saddle-Node (SN)

behavior (Figs. 6b,c). In this work we have focused on the analysis of the period one and period two orbits, and, therefore, we have not continued the branches emanating from the period two solutions. However, the general framework to understand the 2:1 bifurcations will allow us to explain the reason for this wide range of high-order periodicities.

It is interesting to study under which conditions these higher periodic orbits appear. At large values of the inactivation (see Fig. 7d), the region of irregular dynamics disappears, and the period two branch is stable in the whole range between the two period doubling bifurcations. Decreasing inactivation, the period two orbit becomes unstable at lower stimulation periods (Fig. 7c). For still lower inactivation, the first instability becomes subcritical (Fig. 7a, b). It is only when inactivation is reduced (see, for instance, Fig. 7a), that both the period one and the period two solutions become unstable through saddle-node bifurcations as the period $T_{\text {period }}$ is reduced. 

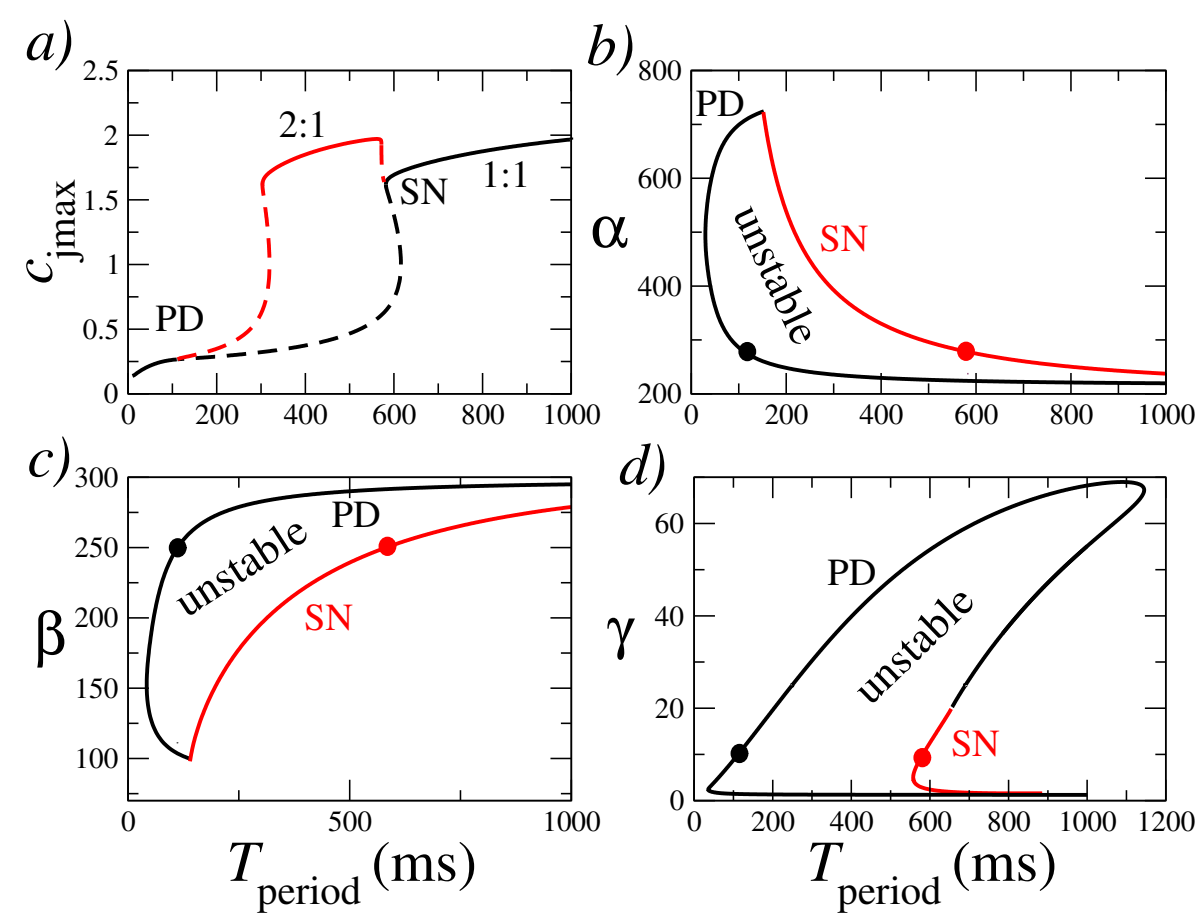

Figure 8. a) Bifurcation diagram depending on period for values of the parameters: $\alpha=278, \beta=250$ and $\gamma=10$. Panels b), c) and d) show the bifurcation lines corresponding to the variation of the SN and PD with parameters $\alpha, \beta$ and $\gamma$. The red and black dots correspond to the position of the saddle-node (SN) and the period-doubling (PD) in the bifurcation diagram in a). The values between the two lines corresponds to the region where the period one orbit is unstable.

\section{Role of diffusion and release strength in the appearance of alternans}

We proceed to analyse now how the parameters describing diffusion and release strength affect the appearance of alternans. For that, we have continued in parameter space the two bifurcation points that limit the stability of the period one orbit. With this process of continuation we obtain the general picture of how the global structure of the bifurcation space is and, more specifically, whether the parameter range which presents alternation is broad or small. In Fig. 8a we show a typical bifurcation diagram, for $\gamma=10$, where the stable period one orbit is limited by a saddle-node $(\mathrm{SN})$ bifurcation at large $T_{\text {period }}$ and a period-doubling (PD) at low $T_{\text {period }}$. Taking the value of $T_{\text {period }}$ at which these limit points appear we follow PD and SN as we change the period and the strength of the release $\alpha$. The resulting curves are shown in Fig. 8b. The region in $T_{\text {period }}-\alpha$ space that presents alternation is clearly limited by the two curves. We observe that very large values of $\alpha$ 

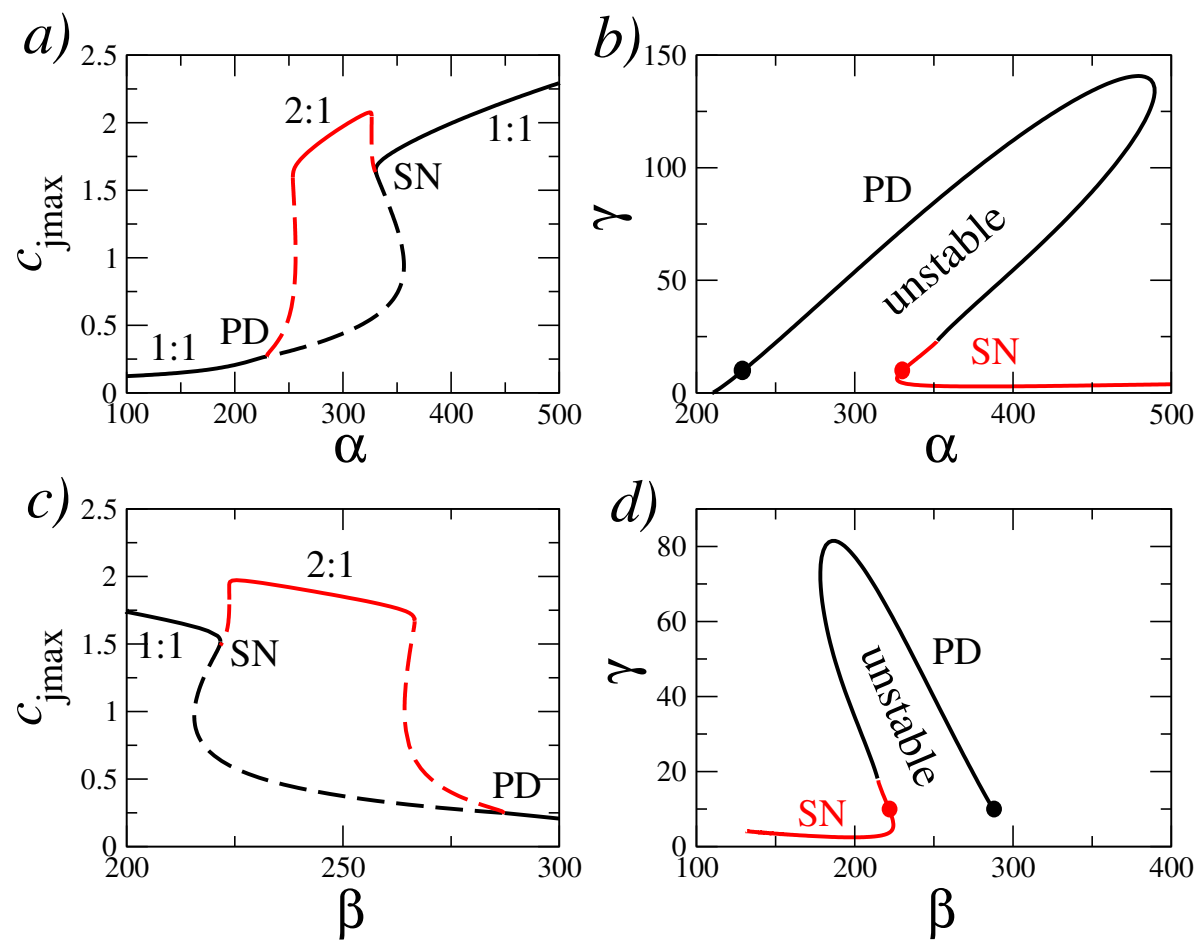

Figure 9. Panels a) and c) show the bifurcation diagram depending on strength of the release $\alpha$ and the diffusion parameter $\beta$ for a $400 \mathrm{~ms}$ stimulation period. Panels b) and d) show the diagram of the continuation bifurcation points $\mathrm{SN}$ and $\mathrm{PD}$ as a function of the inactivation parameter $\gamma$.

precludes the presence of alternans. There are clearly optimal values of the release around $\alpha \simeq 300$ which favour the presence of alternans for a wide range of pacing frequencies.

The effect of diffusion is presented in Fig. 8c. Just as in the case of release strength, there is an optimal value for diffusion at around $\beta=250$ which facilitates alternation for a wide range of pacings. It is quite interesting that the phase diagram with $\beta$ (Fig. 8c) is inverted with respect to that varying $\alpha$ (Fig. 8b). In this case large values preclude alternation altogether, while for smaller values, the parameter space with alternans shrinks becoming smaller and smaller until it finally disappears as the PD collides with the SN.

For completeness, we also present the phase diagram using the parameter $\gamma$, that measures the importance of inactivation, reobtaining again the results $i^{20}$ where the presence of alternans is linked to the time scale of the recovery. As explained before, the mechanism for alternans requires the interaction between the time scale fixed by $\gamma$ and the nonlinear release. It is thus expected that alternation occurs always around periods fixed by the time scale given by $\gamma$. 
We can follow up this analysis by, instead of choosing the stimulation period as bifurcation parameter, choose the strength of release $\alpha$ or diffusion $\beta$ at a given pacing. We take $T_{\text {period }}=400 \mathrm{~ms}$ as the standard period where one should expect alternans. The reasoning behind this selection of phase space cuts is clear. While the role of inactivation is understood, how the possible existence of alternation is affected by diffusion or release strength remains to be addressed.

Regarding the appearance of complex solutions when we vary the strength of the release, $\alpha$ (Fig. 9a), we get the same kind of behaviour as before, where for large and small values of these parameters the period one solution is stable and there are intermediate values where alternans (and more complex behaviour) appear. The same structure is again obtained when the bifurcation parameter is the diffusion, $\beta$ (Fig. 9c). In both cases, the transition is also asymmetrical for large and small values of $\alpha$ and $\beta$. At large $\alpha$ (resp. small $\beta$ ) instability of the period one solution gives rise to alternans (stable period two branch), that then become unstable resulting in complex behaviour before the period one solution regains stability in a period doubling bifurcation.

We can then proceed with the continuation of the bifurcation points that limit the stability of the period one orbit, in order to construct the $\gamma-\alpha$ and $\gamma-\beta$ phase diagrams (Figs. 9b and d). In both cases there is a large range of parameters where alternation occur. Interestingly, the region of instability seems to follow a line in $\gamma-\alpha$ or $\gamma-\beta$ space. However, while it moves to large values of $\alpha$ as $\gamma$ is increased, it is much less dependent on $\beta$. For a broad range of release strengths $(\alpha)$ it is possible to find a corresponding inactivation $\gamma$ that allows for the presence of alternans, although once $\gamma$ is fixed, the $\alpha$ range is limited. This is not the case for diffusion where the global scope is reduced. The scope of values where diffusion can present alternans is sligthly more limited but, more importantly, quite independent on the inactivation $\gamma$. Thus, release strength can be tuned as a function of the recovery levels, but diffusion must be fixed around certain values in order to have alternation. Characteristic time scales of the diffusion, which are normally related with the presence of large proteins and buffers, can have a crucial role in the tendency to generate alternans. This may have important implications for understanding and controlling alternation since diffusion has already been set as a possible target to eliminate the coordination of alternans $\left(\mathrm{see}^{22}\right)$. Here, the diffusion constraints to obtain alternans can present an opportunity to prevent even the apperance of alternation at the local level. 

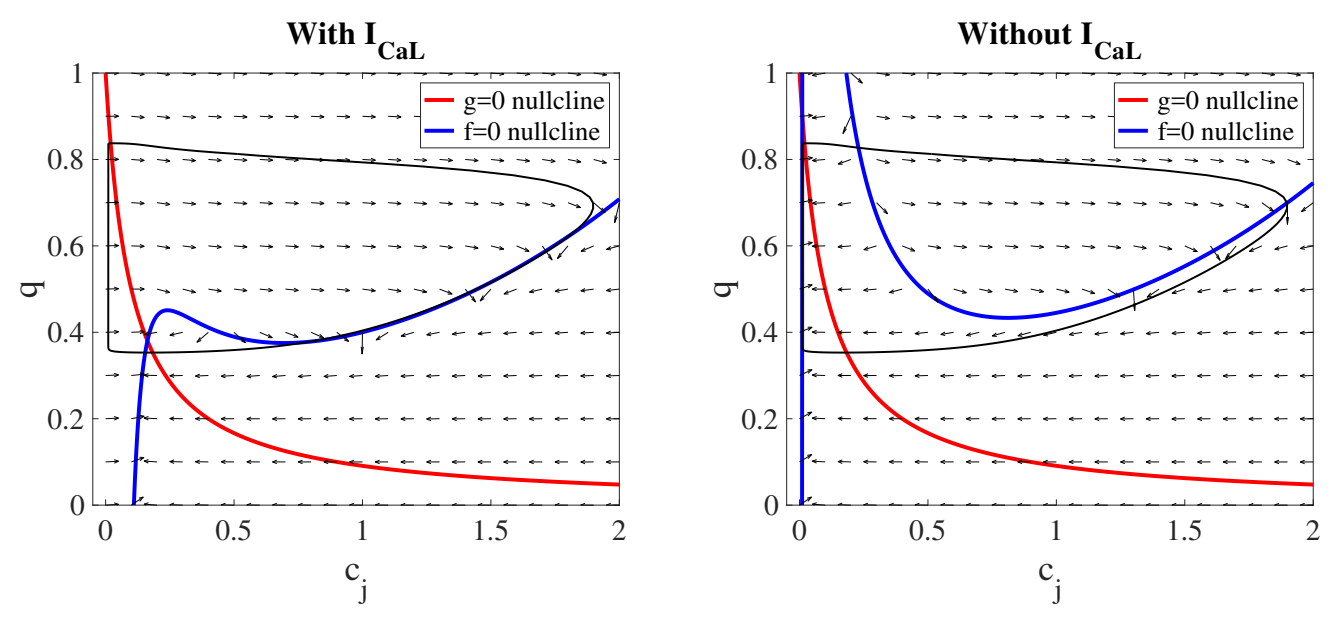

Figure 10. Nullclines corresponding to $\frac{d q}{d t}=g\left(c_{j}, q\right)=0$ in red and $\frac{d c_{j}}{d t}=f\left(q, c_{j}, I_{C a L}\right)=0$ in blue and the flow direction. a) $I_{C a L} \neq 0$, b) $I_{C a L}=0$.

\section{Nullclines and phase diagrams}

A better understanding of the onset of alternans in Eqs. (14), (15) can be obtained studying the nullclines and the corresponding trajectories in phase space. The nullclines, corresponding to $d c_{j} / d t=f\left(q, c_{j}, I_{C a L}\right)=0$ and $d q / d t=g\left(q, c_{j}\right)=0$ give the curves:

$$
q=\frac{\left[\beta\left(c_{j}-c_{0}\right)-I_{C a L}^{\max }\right]\left(1+c_{j}^{2}\right)}{\alpha c_{j}^{2}\left(c_{S R}-c_{j}\right)} \text { and } q=\frac{1}{1+\gamma c_{j}}
$$

where the first curve sets the excitability threshold and the second one the termination of release (Fig. 10b). These curves intersect at a (locally) stable fixed point. When $I_{C a L}^{\max }=0$ (Fig. 10b) the fixed point is located at low values of $c_{j}$. However, when the LCC becomes active, this fixed point changes position, moving to lower values of $q$ and a larger value of $c_{j}$ (Fig. 10a). Not only the fixed points are affected, the velocity flow in phase space changes. The trajectory, shown in black in Fig. 10, is affected consecutively by both phase space flows. First, during a brief period of time $\Delta t$, the nullclines with $I_{C a L}^{\max }$ determine the flow. The final point when $I_{C a L}$ closes can end up at the right or at the left of the nullcline $f\left(q, c_{j}, 0\right)=0$. If to the right, then the trajectory gives an excursion (a calcium transient) before returning to the fixed point (Fig 10b). On the other hand, if the final point is on the left of the nullcline there is no large excursion but a short return to the stable fixed point which was lost for a short time.

Let us consider that the system starts at the position of the fixed point when $I_{C a L}=0$. When the $I_{C a L}$ switch on, the system will move from this point. Assuming that inactivation 
Mechanism for alternans 2:1
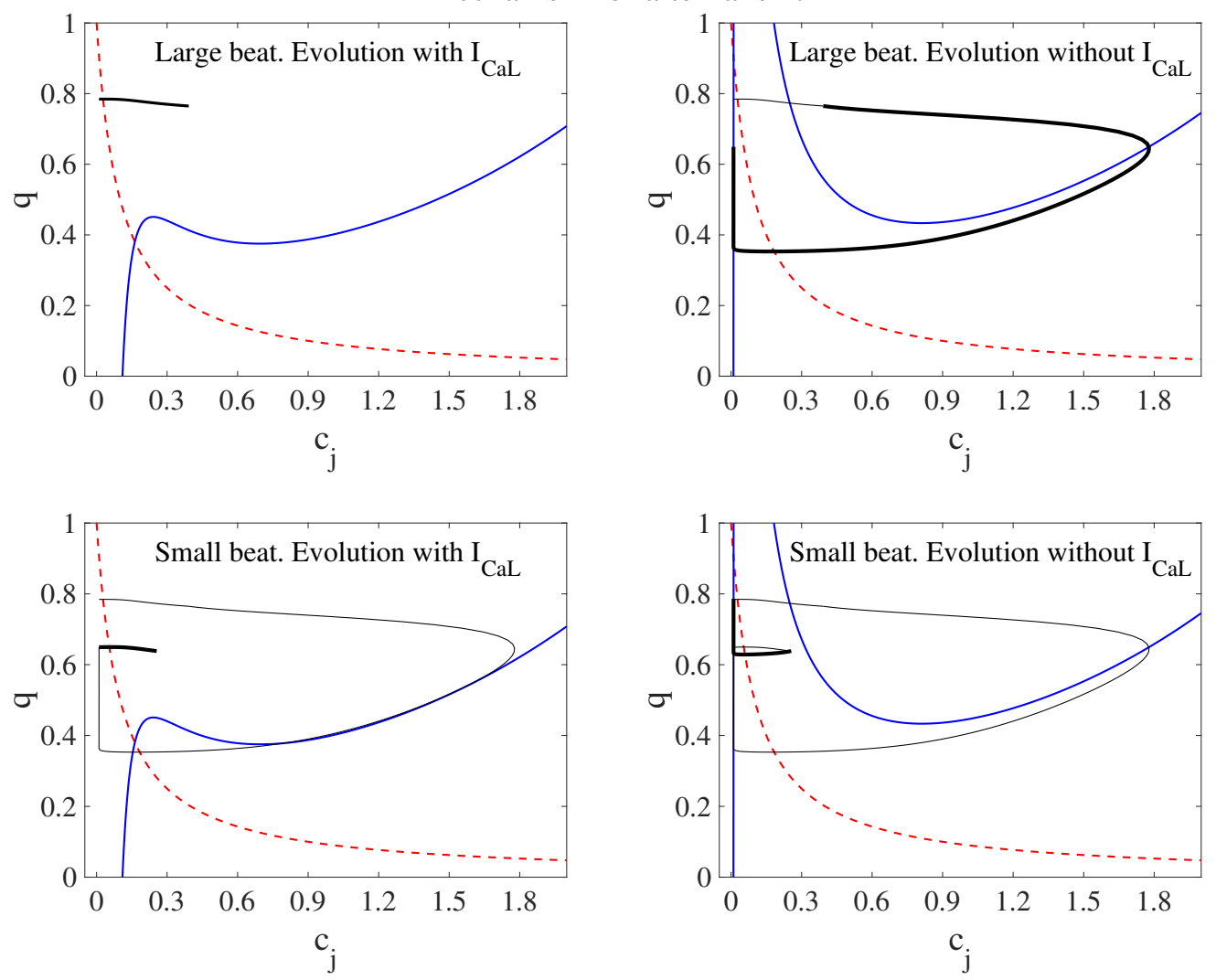

Figure 11. Mechanism for period doubling bifurcation, from period one 1:1 rhythm to 2:1 alternation. a) Trajectory for the first $10 \mathrm{~ms}$ with the corresponding nullclines in blue and red, b) the rest of the trajectory up to a period of $400 \mathrm{~ms}, \mathrm{c})$ and d) same for the next beat.

is slow compared with the time scale $\Delta t$, in which the external current $I_{C a L}$ is acting, we can consider that $q$ is almost constant during this first stage. Then, integrating Eq. (14) it is possible to compute an estimate of the threshold value of $q_{t h r}$ above which the trajectory crosses the nullcline $f\left(q, c_{j}, 0\right)=0$ (see Appendix B):

$$
q_{t h r}=\frac{\beta^{2}}{4 I_{C a L} \alpha c_{S R}}\left[1+\left(\frac{2 \pi}{4+\beta \Delta t / \tau}\right)^{2}\right]
$$

The existence of this limiting value makes the dependence of the trajectory with $q$ very nonlinear. During alternans, one has to expect that the crossing of the nullcline happens only at non-consecutive beats. A clear depiction of this process is sketched in Fig. 11. In one beat the number of recovered RyR2s is at a high value of $q$, a value large enough so the trajectory starting at that point crosses the excitability threshold. If the period is not large 

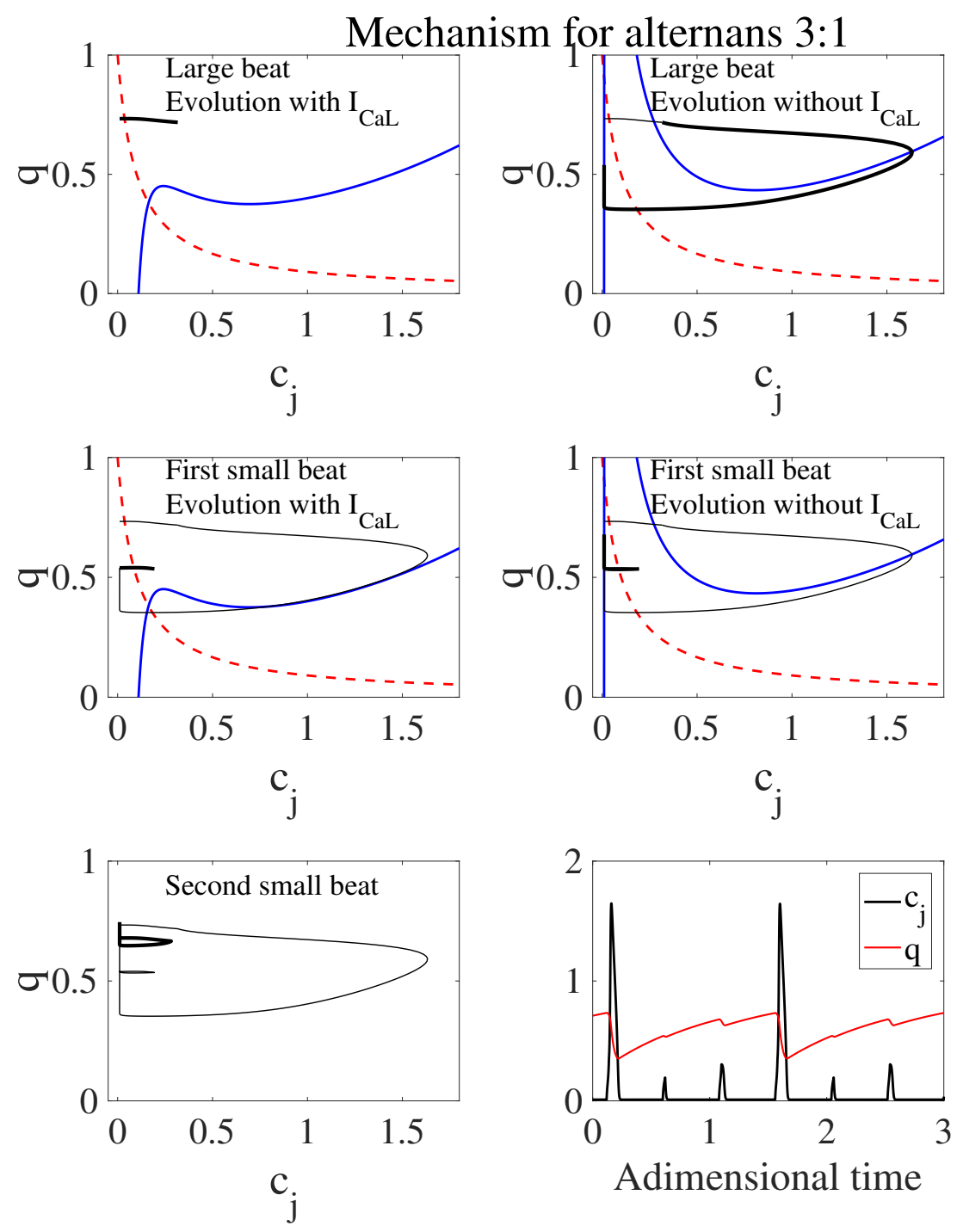

Figure 12. Example of 3:1 periodicity for $T_{\text {period }}=240 \mathrm{~ms}$.

enough, however, by the time the next stimulation arrives, the number of recovered RyR2s $q$ is at a much lower value than before. Then, in this case, the nullcline $f\left(q, c_{j}, 0\right)=0$ is further to the right in phase space and the time $\Delta t$ is too short to increase the calcium level beyond the nullcline. The system, consequently, returns to the fixed point, resulting in a short transient. At the next beat, however, the value of $q$ has recovered enough so as to cross the threshold generating the well-known 2:1 alternans.

From Eq. (17) it is clear that diffusion and release strength play a very important role in 


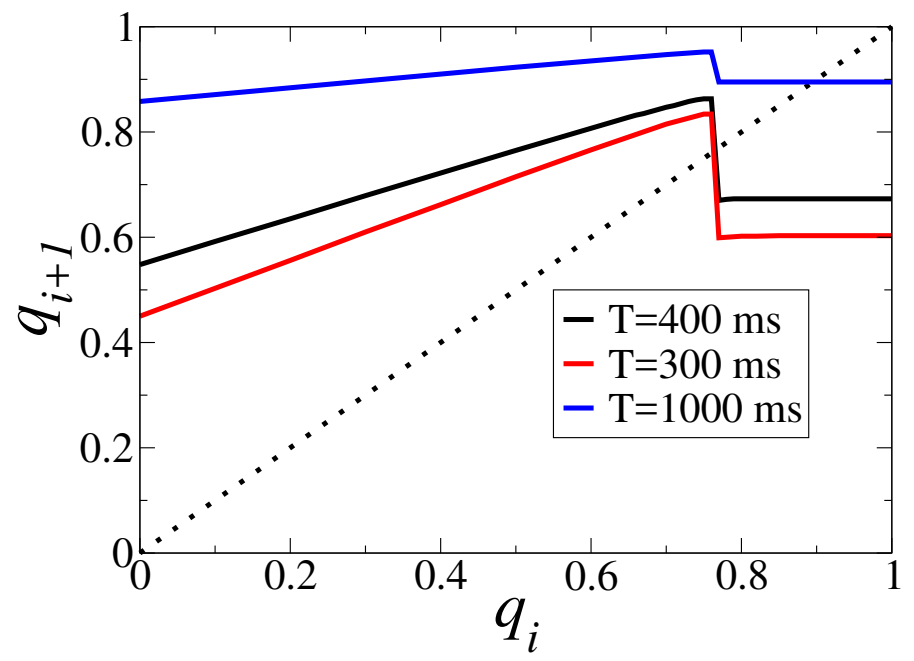

Figure 13. Iterative map $q_{n+1}=f\left(q_{n}\right)$ for periods of $1000 \mathrm{~ms}$ (blue), $400 \mathrm{~ms}$ (black), $300 \mathrm{~ms}$ (red). The dashed line corresponds to $q_{n+1}=q_{n}$

the nature of the bifurcation. They move the limit of excitability. Once the recovery is fixed ( $\gamma$ fixed), the slope of the nullcline determines how a decrease in $q$ affects the ability to go beyond the excitability threshold. This slope depends on the ratio $\beta / \alpha$. However, this same ratio is not present in the determination of the limiting value $q_{t h r}$ leading to a qualitatively different role for diffusion and for the release strength.

A detailed representation of the general process that explains high periodicities is given for a 3:1 rhythm in Fig. 12. If the period of stimulation is further decreased, even after a second short transient, the number of RyR2s has not recovered enough and one has to wait to a third beat (or more beats) in order to be able to elicit a large transient. This mechanism can be generalized to higher periodicities. The existence of this well define threshold $q_{t h r}$ for the onset of a spike, makes it possible to obtain the higher order periodicity and irregular behaviour.

This explanatory picture of the system can be confirmed constructing a return map for the variable $q, q_{n+1}=f\left(q_{n}\right)$ (Fig. 13). Alternans then appear when the slope of the map is smaller than minus one $f^{\prime}<-1$. Higher periodicity could be understood studying higher order maps $q_{n+2}=g\left(q_{n}\right)$. In the maps in Fig. 13 the straight line at low $q$ corresponds to short calcium transients where during the excursion the value of $q$ remains rather constant. One can then obtain the map integrating Eq. (15) with initial value $q_{n}$ for a determined period and setting $c_{j} \simeq 0$. This gives a value of $q_{n+1} \approx 1-\left(1-q_{n}\right) \exp \left(-T_{\text {period }} / \tau\right)$, which 
results in a linear dependence of $q_{n+1}$ with $q_{n}$, as seen in Fig. 13 for different pacings $T_{\text {period }}$. On the other hand, at large $q_{n}$ one obtains an almost constant value of $q_{n+1}$, independent of $q_{n}$. This corresponds to the large spikes where the trajectory rapidly follows the $f=0$ nullcline down to its minimum value, where it jumps to the branch near $c_{j} \simeq 0$, losing the information of the initial state, and ending always approximately at the same value of $q$. The transition between these two branches occurs at a value of $q \simeq q_{t h r}$. Alternans and more complex rhythms appear as intermediate regimes when the system does not stabilize in neither of these two states.

\section{DISCUSSION AND CONCLUSIONS}

In this paper we have presented a simplified model of calcium release which reproduces calcium alternas due to refractoriness. Specifically, we introduce refractoriness as due to inactivated states of the RyR2. Although there is debate on whether these inactivates states actually exist $^{29}$ there are clear indications that some kind of termination or refractoriness

similar to inactivation is present, specially in physiological conditions in heart tissue ${ }^{30}$. The model can thus be considered as a phenomenological description that contains key physiologically relevant features of RyR2 release.

The simplified model can also be considered from two different points of view. Either as a reduction of a whole cell-model where the calcium concentration in each compartment corresponds to average values on the cell, or to the deterministic limit of a CaRU, where the stochastic dynamics of the RyRs has been neglected. In the first type of models, alternation appears, in the mathematical sense, as a period doubling bifurcation of the calcium transient from 1:1 to 2:1 periodicity, presenting sometimes, and under some pacing, different transitions to higher order periodicities. On the other hand, in models with subcellular structure, alternans appears as the coordination of local random sequences of release, nonrelease events. In this latter case, close to the alternans transition each RyR2 cluster has a tendency to generate local alternation, but given the stochastic nature of this behavior, this alternation changes phases continually. Alternans appear in this case as an order-disorder transition (see $\operatorname{Ref}^{22}$ for details). The deterministic limit of the CaRU gives an idea of when local units will present long sequences of release/non-release events.

Fixing cytosolic cacium level is a very reasonable approach if we understand this model as 
the deterministic limit of a RyR2 cluster. Near the cluster, calcium concentration is believed to increase above $100 \mu \mathrm{M}$ while buffers keep cytosolic and subsarcolemma concentration at around 1-10 $\mu \mathrm{M}$ even during the transient making that, from the point of view of the dyadic space, calcium concentration in the cytosol is constant. If we have the whole cell model in mind, it allows us to focus on the effects of refractoriness, discounting the effects of the SERCA pump which might give rise to alternans by a different mechanism related to the presence of high nonlinearities in release as a function of SR content. Following this idea, a constant $I_{C a L}$ current reproduces the current due to the opening of a LCC channel and, thus, it is a good representation of what occurs in a CaRU. In whole cell models, however, the $I_{C a L}$ current results of the sum of thousands of LCC currents and presents a very different form. An important effect that we are not taking into account in this case, is the feedback between calcium concentration and L-type calcium current, due to calcium induced inactivation of the latter. However, even if peak $I_{C a L}$ alternations have been observed in experiments ${ }^{31}$, we argue that they are subsequent to junctional calcium alternations.

Another important simplification of the model, i.e., fixing the SR concentration, simplifies the homestatic global effects seen in whole-cell models and allows us to differentiate alternans due to SR release refractoriness from those due to SR content alternations. We acknowledge that this approximation is, however, not straightforward from the point of view of the deterministic limit of the RyR2 cluster. Given that SR calcium levels drop locally in the part of the SR close to the ryanodine receptor, a more appropriate approximation to eliminate homeostatic calcium levels, regulated by the interaction of SERCA pump and exchanger with the RyR2 function, would be to consider a second calcium concentration describing the SR junctional calcium drop while, effectively, the overall SR concentration remains constant. However, we have found in our preliminary tests that this extra description does not provide any new basic insight to the problem as long as the cluster of receptors has a significant inactivation. The drop of calcium levels at the junctional SR only has relevance when calcium release is terminated because calcium depletes in this area. Given that this situation is not the one we address here, we have simplified the model as much as possible.

The results of the model can now be analyzed having in mind this double perspective. The model reproduces the finding of ${ }^{20}$ where inactivation was key to observe alternans due to SR release refractoriness. The general idea behind the mechanism is sustained in our analysis. However, it provides further light on the effects of release strength and local diffusion. While 
it is possible to find a broad range of release strengths where alternation appears provided the time scale fixed by recovery is adequate, such versatility is not present for diffusion, that can not change as much once release strenght is fixed. This points to a key role of diffusion in the generation of alternans. This is particularly important if we take the image of the model as the deterministic limit of a cluster of RyR2. In subcellular stochastic models, a change in diffusion has been shown to eliminate alternans by disrupting the coordination of the local alternations. Our results hints at the possibility that changing diffusion might also be affecting the same generation of local alternate events. A lower diffusion might not only disrupt coordination by reducing the coupling among CaRU, but it would also reduce the average persistence of the local alternation.

Analysis of the model has also helped to unveal the origin of the non-linear nature of the alternans due to SR release refractoriness, which explains the appearance not only of period doubling bifurcations but of higher order periodicities. We have found that there is a strongly nonlinear dependence of calcium release with the level of recovered receptors, which is linked to the excitable gap defined by the nullclines of the model. There is a critical value of the number of recovered receptors that leads to a large calcium release, which gives rise to an on/off process. Close to the critical value, the system can easily transit from release to non-release events. This can lead to high order periodicity and quasi-intermittencies. Still, in a real cell stochasticity would probably make higher order periodicities non-observable.

The model introduced and analyzed here, thus, provides key insights as to the mechansim and effects of SR release refractoriness in whole-cell models and subcellular description, explaining why RyR2 clusters in real cells have this strong tendency to produce this crucial pro-arrythmic feature in cell signalling.

\section{ACKNOWLEDGEMENTS}

We thank L. Hove-Madsen and Y. Shiferaw for fruitful discussions. The authors acknowledge financial support from Fundació La Marató de TV3 and from the Spanish Ministerio de Economía y Competitividad (MINECO) under grant number SAF2014-58286-C2-2-R and FIS2015-66. 


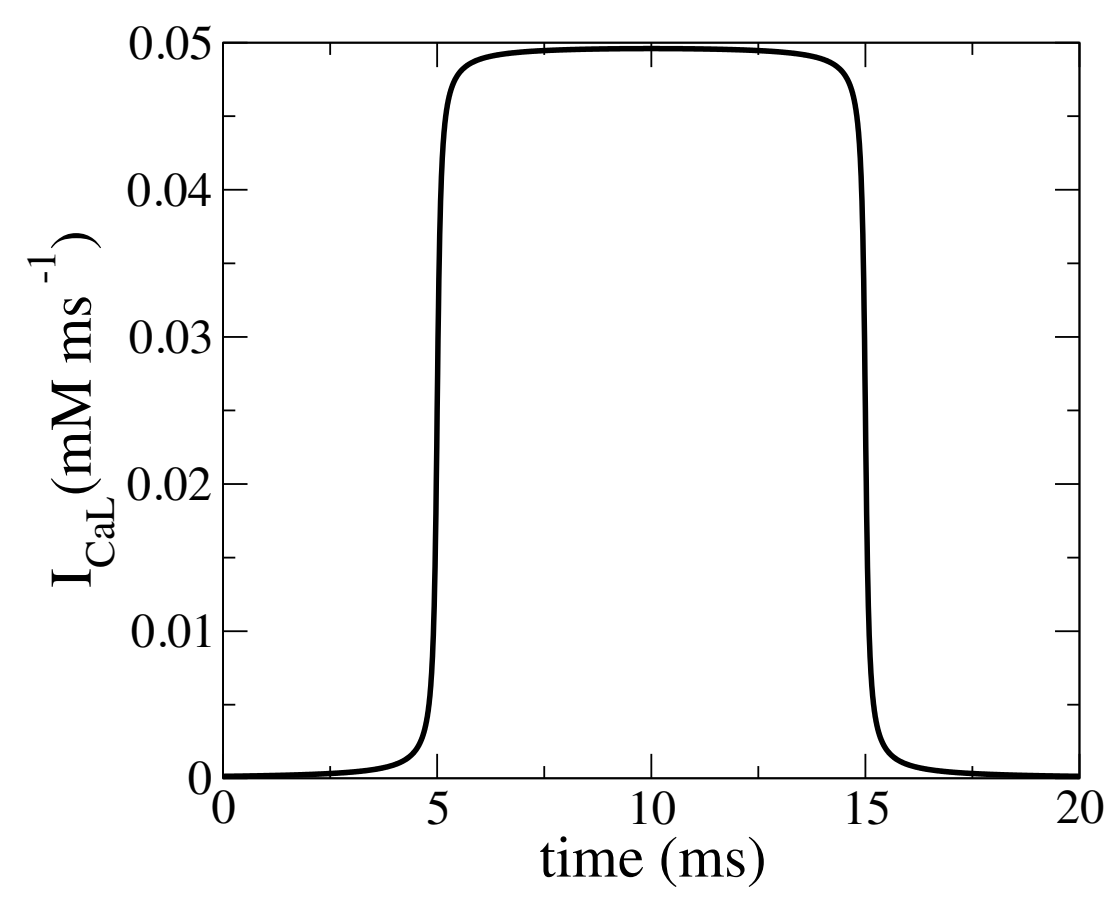

Figure 14. Calcium pulse $I_{C a L}(t)$ obtained from Eq. (A1)

\section{Appendix A: Continuation method}

In order to reduce Eqs. (14), (15) to an autonomous problem that can be analyzed by AUTO, we rewrite the forcing in terms of the periodic solutions of an auxiliary autonomous ordinary differential equations (ODE) system ${ }^{27,32}$. We represent the calcium current $I_{C a L}$ as a pulse of duration $\Delta t=10 \mathrm{~ms}$, that starts with a delay $t_{c 1}$ after each $T_{\text {period }}$ and with a halftime steep value, $m$, to its maximum value $I_{\text {CaLmax }}$ (Fig. 14). We adjust the parameters in order to ensure a value of $I_{C a L m a x} \simeq 30 \mu \mathrm{M} / \mathrm{s}$. The expression for $I_{C a L}$ then is

$$
I_{C a L}(t)=\frac{I_{C a L \max }}{\pi}\left\{0.5+\arctan \left[A h\left(x_{s}, y_{s}\right)\right]\right\}
$$

with

$$
\begin{gathered}
h\left(x_{s}, y_{s}\right)=y_{s} \cos \left(\frac{2 \pi t_{c 1}}{T_{\text {period }}}\right)+x_{s} \sin \left(\frac{2 \pi t_{c 1}}{T_{\text {period }}}\right)-\cos \left(\frac{\pi \Delta t}{T_{\text {period }}}\right) \\
A=\frac{m T_{\text {period }}^{2}}{2 \pi \Delta t}
\end{gathered}
$$

and $x_{s}$ and $y_{s}$ periodic functions of time solution of the equations:

$$
\begin{aligned}
& \frac{d x_{s}}{d t}=x_{s}+\left(2 \pi / T_{\text {period }}\right) y_{s}-x_{s}\left(x_{s}^{2}+y_{s}^{2}\right) \\
& \frac{d y_{s}}{d t}=y_{s}-\left(2 \pi / T_{\text {period }}\right) x_{s}-y_{s}\left(x_{s}^{2}+y_{s}^{2}\right)
\end{aligned}
$$




\section{Appendix B: Threshold value $q_{t h r}$}

To calculate the threshold value $q_{t h r}$ we can integrate Eq. (14) from the value $c_{j} \simeq 0$ to the value of $c_{j}$ at the nullcline $f\left(q, c_{j}, 0\right)=0$. Neglecting terms of order $c_{j}^{3}$ (and $\left.c_{0}\right)$, then Eq. (14) becomes

$$
\frac{d c_{j}}{d t}=\alpha q c_{S R} c_{j}^{2}-\beta c_{j}+I_{C a L}
$$

Considering $q$ constant, and integrating, we obtain:

$$
\int_{0}^{\beta / \alpha q c_{S R}} \frac{d c_{j}}{\alpha q c_{S R} c_{j}^{2}-\beta c_{j}+I_{C a L}}=\int_{0}^{\Delta t / \tau} d t
$$

that results in:

$$
\frac{4}{\Delta} \arctan \frac{\beta}{\Delta}=\frac{\Delta t}{\tau}
$$

with $\Delta=\sqrt{4 I_{C a L} \alpha c_{S R} q-\beta^{2}}$. When diffusion is not too small a good approximation for $q_{t h r}$ can be obtained expanding the arctan as

$$
\frac{4}{\Delta} \arctan \frac{\beta}{\Delta} \simeq \frac{4}{\Delta}\left(\frac{\pi}{2}-\frac{\Delta}{\beta}\right)=\frac{\Delta t}{\tau}
$$

so

$$
q_{t h r}=\frac{\beta^{2}+\left(\frac{2 \pi}{\Delta t / \tau+4 / \beta}\right)^{2}}{4 I_{C a L} \alpha c_{S R}}
$$

For the values used in the paper it gives a value of $\Delta=174.5$ and a value of the inactivation threshold $q_{\text {thres }}=0.67$.

\section{REFERENCES}

${ }^{1}$ J.M. Smith, E.A. Clancy, C.R. Valeri, J.N. Ruskin, R.J. Cohen, "Electrical alternans and cardiac electrical instability", Circulation 77, 11021 (1988).

${ }^{2}$ J.B. Nolasco \& R.W. Dahlen, "A graphic method for the study of alternation in cardiac action potentials", J. Appl. Physiol. 25, 191196 (1968).

${ }^{3}$ E. Chudin, J. Goldhaber, A. Garfinkel, J. Weiss, \& B. Kogan, "Intracellular Ca2+ dynamics and the stability of ventricular tachycardia", Biophysical journal 77, 2930-2941 (1999).

${ }^{4}$ B. Echebarria, E. Alvarez-Lacalle, I.R. Cantalapiedra, A. Peñaranda, "Mechanisms Underlying Cardiac Alternans Electro-Mechanical Cardiac Alternans", 113-128, Springer International Publishing, 2016. 
${ }^{5}$ Z. Qu, M. Nivala, \& J.N. Weiss, "Calcium alternans in cardiac myocytes: order from disorder", Journal of molecular and cellular cardiology 58, 100-109 (2013).

${ }^{6}$ J.N. Edwards \& L.A. Blatter, "Cardiac alternans and intracellular calcium cycling", Clinical and Experimental Pharmacology and Physiology 41, 524-532 (2014).

${ }^{7}$ M.E. Díaz, S.C. O’Neill, D.A. Eisner, "Sarcoplasmic reticulum calcium content fluctuation is the key to cardiac alternans", Circulation Research 94, 650-656 (2004).

${ }^{8}$ Y. Shiferaw, M.A. Watanabe, A. Garfinkel, J.N. Weiss, \& A. Karma, "Model of intracellular calcium cycling in ventricular myocytes", Biophysical journal 85, 3666-3686 (2003).

${ }^{9}$ E. Picht, J. DeSantiago, L. Blatter, D. Bers, "Cardiac alternans do not rely on diastolic sarcoplasmic reticulum calcium content fluctuations". Circulation Research 99, 740-748 (2006).

${ }^{10}$ J. Hüser, Y.G. Wang, K.A. Sheehan, F. Cifuentes, S.L. Lipsius, L.A. Blatter, "Functional coupling between glycolysis and excitation-contraction coupling underlies alternans in cat heart cells", The Journal of Physiology 524, 795-806 (2000).

${ }^{11}$ L. Wang, R.C. Myles, N.M. De Jesus, A.K. Ohlendorf, D.M. Bers, C.M. Ripplinger, "Optical mapping of sarcoplasmic reticulum $\mathrm{Ca} 2+$ in the intact heart: ryanodine receptor refractoriness during alternans and fibrillation", Circulation Research 114, 1410-1421 (2014).

${ }^{12}$ V.M. Shkryl, J.T. Maxwell, T.L. Domeier, and L.A. Blatter, "Refractoriness of sarcoplasmic reticulum Ca release determines Ca alternans in atrial myocytes" Am J Physiol Heart Circ Physiol 302, H2310-20 (2012).

${ }^{13}$ J.G. Restrepo, J.N. Weiss, \& A. Karma, "Calsequestrin-mediated mechanism for cellular calcium transient alternans", Biophysical journal 95, 3767-3789 (2008).

${ }^{14}$ C.A. Lugo, I.R. Cantalapiedra, A. Peñaranda, L. Hove-Madsen, B. Echebarria, "Are SR Ca content fluctuations or SR refractoriness the key to atrial cardiac alternans?: insights from a human atrial model", American Journal of Physiology - Heart and Circulatory Physiology 306, H1540-H1552 (2014)

${ }^{15}$ K.C. Chang, J.D. Bayer, N.A. Trayanova, "Disrupted calcium release as a mechanism for atrial alternans associated with human atrial fibrillation", PLoS Comput Biol. 10, e1004011 (2014).

${ }^{16}$ X. Cui, R.J. Rovetti, L. Yang, A. Garfinkel, J.N. Weiss \& Z. Qu, "Period-doubling bifurcation in an array of coupled stochastically excitable elements subjected to global periodic forcing", Physical Review Letters 103, 044102 (2009). 
${ }^{17}$ R. Rovetti, X. Cui, A. Garfinkel, J.N. Weiss, \& Z. Qu, "Spark-induced sparks as a mechanism of intracellular calcium alternans in cardiac myocytes", Circulation Research 106, 1582-1591 (2010).

${ }^{18}$ M. Nivala \& Z. Qu, "Calcium alternans in a couplon network model of ventricular myocytes: role of sarcoplasmic reticulum load", American Journal of Physiology-Heart and Circulatory Physiology 303, H341-H352 (2012).

${ }^{19}$ Z. Qu, M.B. Liu, \& M. Nivala, "A unified theory of calcium alternans in ventricular myocytes", Scientific Reports 6, 35625 (2016).

${ }^{20}$ E. Alvarez-Lacalle, I.R. Cantalapiedra, A. Peñaranda, J. Cinca, L. Hove-Madsen, B. Echebarria, "Dependency of Calcium Alternans on Ryanodine Receptor Refractoriness", PLoS ONE 8, e55042 (2013).

${ }^{21}$ T.R. Shannon, F. Wang, J. Puglisi, C. Weber, D.M. Bers, "A mathematical treatment of integrated Ca dynamics within the ventricular myocyte", Biophys J 87, 3351-3371 (2004).

${ }^{22}$ E. Alvarez-Lacalle, B. Echebarria, J. Spalding, Y. Shiferaw, "Calcium Alternans is Due to an Order-Disorder Phase Transition in Cardiac Cells", Physical Review Letters 114, 108101 (2015).

${ }^{23}$ I.R. Cantalapiedra, C. Lugo, A. Peñaranda, B. Echebarria, "Calcium alternans produced by increased Sarcoplasmic Reticulum Refractoriness", Computers in Cardiology 38, 645$648(2011)$.

${ }^{24}$ A. Peñaranda, E. Alvarez-Lacalle, I.R. Cantalapiedra, B. Echebarria, "Nonlinearities due to Refractoriness in SR Ca Release", Computers in Cardiology 39, 297-300 (2012).

${ }^{25}$ M.D. Stern, L.-S. Song, H. Cheng, J.S.K. Sham, H.T. Yang, K.R. Boheler, E. Rios, "Local control models of cardiac excitationcontraction coupling: a possible role for allosteric interactions between ryanodine receptors", J. Gen. Phys. 113, 469489 (1999).

${ }^{26}$ J.P. Keener, "Invariant Manifolds Reductions for Markovian Ion Channel Dynamics", Journal of Mathematical Biology 58, 447-457 (2009).

${ }^{27}$ E.J. Doedel, A.R. Champneys, T.F. Fairgrieve, Y.A. Kuznetsov, B. Sandstede, and X. Wang, AUTO97: Continuation and bifurcation software for ordinary differential equations, 1998, http://cmvl.cs.concordia.ca/publications/auto97.ps.gz.

${ }^{28}$ B. Ermentrout, Simulating, Analyzing, and Animating Dynamical Systems: A Guide to XPPAUT for Researchers and Students, SIAM 2002, Philadelphia, USA. 
${ }^{29}$ A. Chu, M. Fill, E. Stefani, M.L. Entman, "Cytoplasmic Ca2+ does not inhibit the cardiac muscle sarcoplasmic reticulum ryanodine receptor Ca2+ channel, although $\mathrm{Ca} 2+$-induced $\mathrm{Ca} 2+$ inactivation of Ca2+ release is observed in native vesicles", J. Membr. Biol. 135, 4959 (1993).

${ }^{30}$ M.D. Stern and H. Cheng, "Putting out the fire: what terminates Calcium-induced Calcium release in cardiac muscle?", Cell Calcium 35, 591601 (2004).

${ }^{31}$ A. Llach, C.E. Molina, J. Fernandes, J. Padro, J. Cinca, L. Hove-Madsen, "Sarcoplasmic reticulum and L-type $\mathrm{Ca}(2)(+)$ channel activity regulate the beat-to-beat stability of calcium handling in human atrial myocytes", J Physiol 589, 3247-62 (2011).

${ }^{32}$ H. Croisier and P. C. Dauby, "Continuation and bifurcation analysis of a periodically forced excitable system", J. Theor. Biol. 246, 430-48 (2007). 